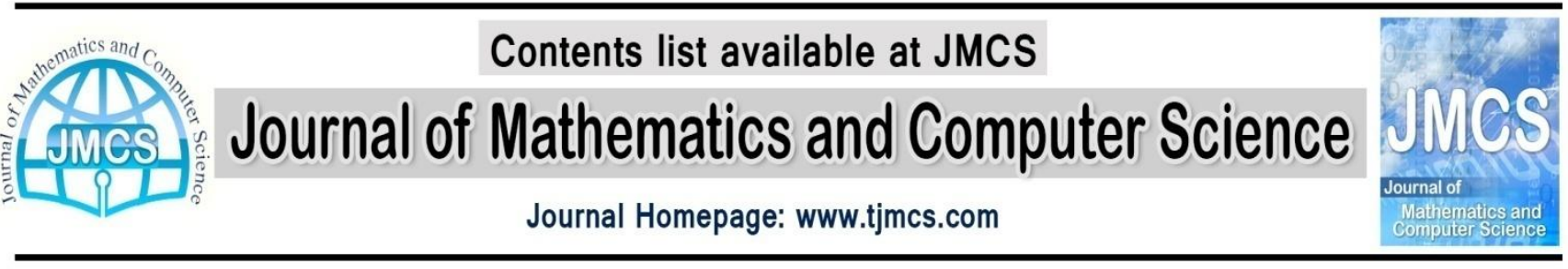

\title{
Numerical method for solving optimal control problem of stochastic Volterra integral equations using block pulse functions
}

\author{
M. Saffarzadeh ${ }^{\mathrm{a}}$, A. Delavarkhalafi ${ }^{\mathrm{a}}$, Z. Nikoueinezhad ${ }^{\mathrm{a}}$ \\ ${ }^{a}$ Department of Mathematics, Yazd University P.O. Box: 89195-741 Yazd, Iran \\ E-mail: m_saffarzadeh2005@yahoo.com
}

Article history:

Received April 2014

Accepted May 2014

Available online June 2014

\begin{abstract}
In this paper, a numerical method for solving a general optimal control of systems is presented. These systems governed by stochastic Volterra integral equations. This method is based on block pulse functions. By using the properties of block pulse functions and associated operational matrices, optimal control problem is converted to an optimization problem and will be solved via mathematical programming techniques. The error estimations and associated theorems have been provided. Finally, some numerical examples are presented to show the validity and efficiency of the proposed method.
\end{abstract}

Keywords: Stochastic Volterra integral equations; Optimal control; Block pulse functions; Stochastic operational matrix.

\section{Introduction}

The theory of optimal control for stochastic differential equations is a mathematical challenging and it has been considered in many fields such as economics, engineering, biology and finance [1]-[7]. The stochastic optimal control problem has been studied in textbooks [8], [9].

In deterministic setting, there are many text books for analytic solutions of optimal control problems such as [8]-[15]. Also, numerical methods for these problems have been provided in [16]-[22].

Stochastic optimal control problems are studied by many researchers[8], [9], [23]. Every system of controlled stochastic differential equations can be usually written by a system of controlled stochastic Volterra integral equations. Øksendal and Zhang have presented a maximum principle for optimal control of general stochastic Volterra equations[24]. However there are few literature to demonstrate some analytical research on the necessary conditions on the existence solution optimal control of stochastic Volterra integral equations [25]-[28].

Except in a limited number of stochastic optimal control problems, most of them do not have an explicit solution. The reason is that they are often dependent on a noise source. Therefore, there 
have been many attempts to develop new methods for obtaining numerical solutions which reasonably approximate the exact solutions.

The Markov chain approximation method for the solution of quite general stochastic control problems was employed by Kushner[29]. Munk discussed about the applicability of the approach to financial control problems and alternative approaches by using the Kushner's method [30], [31]. Chavanasporn and Ewald introduced a numerical method to solve stochastic optimal control problems, which are linear in the control [32]. The aim of this research is to develop a numerical method based on block pulse functions (BPFs) to solve the general optimal control of stochastic Volterra integral equations. One of the standard tools in numerical analysis is approximating a function as a linear combination of a set of orthogonal basis functions. Maleknejad et al. investigated numerical solution of stochastic Volterra integral equations using block pulse functions and their stochastic operational matrix of integration [33]. These matrices are applied to reduce a controlled stochastic integral equation in a system of algebraic equations by expanding the state vector process $\mathrm{X}(\mathrm{t})$ and control vector process $\mathrm{u}(\mathrm{t})$ as BPFs.

The optimal control problem of stochastic integral equations is introduced in section 2. In section 3 , the BPFs, their properties and error estimates theorems are briefly presented. Section 4 is devoted to the solution of optimal control problem of stochastic Volterra integral equations by using the operational matrices of BPFs. Section 5 will present some numerical examples illustrating the efficiency and accuracy of the proposed method. Finally, section 6 gives some brief conclusion.

\section{Preliminaries and formulation}

Let $\left(\Omega, \mathcal{F}, \mathcal{F}_{\mathrm{t}}, \mathrm{P}\right)$ be a filtrated probability space and $\mathrm{B}(\mathrm{t}), \mathrm{t} \geq 0$ is a $\mathcal{F}_{\mathrm{t}}$-measurable real valued Brownian motion. Consider the dynamic optimization problem in which the behavior of dynamic system $x(t)$ at time $t$ is governed by the following controlled stochastic Volterra equation:

$$
\mathrm{x}^{\mathrm{u}}(\mathrm{t})=\mathrm{x}_{0}+\int_{0}^{\mathrm{t}} \mathrm{b}\left(\mathrm{x}^{\mathrm{u}}(\mathrm{s}), \mathrm{u}(\mathrm{s})\right) \mathrm{ds}+\int_{0}^{\mathrm{t}} \sigma\left(\mathrm{x}^{\mathrm{u}}(\mathrm{s}), \mathrm{u}(\mathrm{s})\right) \mathrm{dB}(\mathrm{s}), \mathrm{x}(0)=\mathrm{x}_{0},
$$

where $u(t)$ is our control process. Let $U$ be a closed, convex subset of $\mathbb{R}$, which will be the space of admissible controls.

The goal of the problem is to find $\mathrm{u}(\mathrm{t})$, and then the performance functional of the following form:

$$
J(u)=E\left[\int_{0}^{T} f\left(x^{u}(t), u(t)\right) d s+g\left(x^{u}(T)\right)\right],(2)
$$

is minimized by $\mathrm{u}(\mathrm{t})$, where $\mathrm{b}, \sigma: \mathbb{R} \times \mathrm{U} \rightarrow \mathbb{R}$ and $\mathrm{f}: \mathbb{R} \times \mathrm{U} \rightarrow \mathbb{R}$ are $\mathcal{F}_{\mathrm{t}}$-predictable and $\mathrm{g}: \mathbb{R} \rightarrow \mathbb{R}$ is $\mathcal{F}_{\mathrm{T}}$-measurable. Now, we intend to approximate the stochastic Volterra control problem via block pulse basis functions.

\section{Block-pulse functions (BPFs)}

The block pulse functions from a complete set of orthogonal functions which defined on the interval $[0, \mathrm{~T})$ by

$$
\phi_{\mathrm{i}}(\mathrm{t})= \begin{cases}1, & (\mathrm{i}-1) \mathrm{h} \leq \mathrm{t}<i h \\ 0, & \text { otherwise }\end{cases}
$$


where $\mathrm{i}=1,2, \ldots, \mathrm{m}$ with a positive integer value for $\mathrm{m}$ and $\mathrm{h}=\frac{\mathrm{T}}{\mathrm{m}}$. In this paper, we assume that $\mathrm{T}=1$, so BPFs are defined over $[0,1)$, and $\mathrm{h}=\frac{1}{\mathrm{~m}}$.

\subsection{Properties of BPFs}

Here we review some elementary properties of BPFs [34], [35]. For each $\mathrm{i}, \mathrm{j}=1,2, \ldots, \mathrm{m}$, we have:

Disjointness:

$$
\phi_{\mathrm{i}}(\mathrm{t}) \phi_{\mathrm{j}}(\mathrm{t})= \begin{cases}\phi_{\mathrm{i}}(\mathrm{t}), & \mathrm{i}=\mathrm{j}, \\ 0, & \mathrm{i} \neq \mathrm{j},\end{cases}
$$

Orthogonality:

$$
\int_{0}^{1} \phi_{\mathrm{i}}(\mathrm{t}) \phi_{\mathrm{j}}(\mathrm{t}) \mathrm{dt}=\mathrm{h} \delta_{\mathrm{ij}}
$$

where $\delta_{\mathrm{ij}}$ is Kronecker delta.

\section{Completeness:}

For every $f \in \mathcal{L}^{2}[0,1)$ when $m$ tend to the infinity, Parseval's identity holds:

$$
\int_{0}^{1} \mathrm{f}^{2}(\mathrm{t}) \mathrm{dt}=\sum_{\mathrm{n}=1}^{\infty} \mathrm{f}_{\mathrm{n}}^{2}\left\|\phi_{\mathrm{n}}(\mathrm{t})\right\|^{2},
$$

where

$$
\mathrm{f}_{\mathrm{n}}=\frac{1}{\mathrm{~h}} \int_{0}^{1} \mathrm{f}(\mathrm{t}) \phi_{\mathrm{n}}(\mathrm{t}) \mathrm{dt}, \quad \mathrm{n}=1,2, \ldots
$$

\subsection{The operational matrices of the BPFs}

The operational matrices of integration $\mathrm{P}_{\mathrm{B}}$, stochastic integration $\mathrm{P}_{\mathrm{BS}}$ and product $\widetilde{\mathrm{F}}_{\mathrm{B}}$ respectively are given by the following and details of obtaining these matrices can be found in [33], [36], [37]

$$
\begin{aligned}
& \int_{0}^{\mathrm{t}} \Phi_{\mathrm{m}}(\mathrm{s}) \mathrm{d} \mathrm{s} \simeq \mathrm{P}_{\mathrm{B}} \Phi_{\mathrm{m}}(\mathrm{t}), \\
& \int_{0}^{\mathrm{t}} \Phi_{\mathrm{m}}(\mathrm{s}) \mathrm{dB}(\mathrm{s}) \simeq \mathrm{P}_{\mathrm{BS}} \Phi_{\mathrm{m}}(\mathrm{t}), \\
& \Phi_{\mathrm{m}}(\mathrm{t}) \Phi_{\mathrm{m}}^{\mathrm{T}}(\mathrm{t}) \mathrm{F}=\widetilde{\mathrm{F}}_{\mathrm{B}} \Phi_{\mathrm{m}}(\mathrm{t}),
\end{aligned}
$$

where $\mathrm{B}(\mathrm{s})$ is a Brownian motion process,

$$
\Phi_{\mathrm{m}}(\mathrm{t})=\left(\phi_{1}(\mathrm{t}), \phi_{2}(\mathrm{t}), \ldots, \phi_{\mathrm{m}}(\mathrm{t})\right)^{\mathrm{T}},
$$




$$
\begin{aligned}
F= & \left(f_{1}, f_{2}, \ldots, f_{m}\right)^{T}, \\
P_{B} & =\frac{h}{2}\left(\begin{array}{lllll}
1 & 2 & 2 & \ldots & 2 \\
0 & 1 & 2 & \ldots & 2 \\
0 & 0 & 1 & \ldots & 2 \\
\vdots & \vdots & \vdots & \ddots & \vdots \\
0 & 0 & 0 & \ldots & 1
\end{array}\right), \\
P_{B S} & =\left(\begin{array}{lllll}
p_{11} & p_{12} & p_{13} & \ldots & p_{1 m} \\
p_{21} & p_{22} & p_{23} & \ldots & p_{2 m} \\
p_{31} & p_{32} & p_{33} & \ldots & p_{3 m} \\
\vdots & \vdots & \vdots & \ddots & \vdots \\
p_{m 1} & p_{m 2} & p_{m 3} & \ldots & p_{m m}
\end{array}\right), \\
p_{i j}= & \begin{array}{llll}
\text { B }\left(\frac{1-1}{2} h\right)-B((i-1) h) & i=j, \\
B(i h)-B((i-1) h) & j>i, \\
0 \quad & & & \text { otherwise, }
\end{array}
\end{aligned}
$$

and

$$
\widetilde{\mathrm{F}}_{\mathrm{B}}=\left(\begin{array}{ccccc}
\mathrm{f}_{1} & 0 & 0 & \ldots & 0 \\
0 & \mathrm{f}_{2} & 0 & \ldots & 0 \\
0 & 0 & \mathrm{f}_{3} & \ldots & 0 \\
\vdots & \vdots & \vdots & \ddots & \vdots \\
0 & 0 & 0 & \ldots & \mathrm{f}_{\mathrm{m}}
\end{array}\right) .
$$

\subsection{Function approximation}

A function $f(t)$ defined over the interval $[0,1)$ may be expanded with respect to $\phi_{i}(t), i=1,2, \ldots, m$ as follows:

$$
\mathrm{f}(\mathrm{t}) \simeq \sum_{\mathrm{i}=1}^{\mathrm{m}} \mathrm{f}_{\mathrm{i}} \phi_{\mathrm{i}}(\mathrm{t})=\mathrm{F}^{\mathrm{T}} \Phi_{\mathrm{m}}(\mathrm{t}),
$$

where $F=\left(f_{1}, f_{2}, \ldots, f_{m}\right)^{T}$ and $f_{i}$ are defined by $(7)$.

Theorem 3.1. [37] Suppose that $f(t)$ is an arbitrary real bounded function, which is square integrable in the interval $[0,1)$, and $e(t)=f(t)-\hat{f}_{m}(t), t \in[0,1)$, which $\hat{f}_{m}(t)=\sum_{i=1}^{m} f_{i} \phi_{i}(t)$ is the block pulse series of $f(t)$. Then,

$$
\|\mathrm{e}(\mathrm{t})\| \leq \frac{\mathrm{h}}{2 \sqrt{3}} \sup _{\mathrm{t} \in[0,1)}\left|\mathrm{f}^{\prime}(\mathrm{t})\right|
$$

Similarly, a two dimensional function $\mathrm{k}(\mathrm{t}, \mathrm{s}) \in \mathcal{L}^{2}([0,1) \times[0,1))$ can be expand into BPFs by 


$$
\mathrm{k}(\mathrm{t}, \mathrm{s}) \simeq \sum_{\mathrm{i}=1}^{\mathrm{m}} \sum_{\mathrm{j}=1}^{\mathrm{m}} \mathrm{k}_{\mathrm{ij}} \phi_{\mathrm{i}}(\mathrm{t}) \phi_{\mathrm{j}}(\mathrm{s})=\Phi_{\mathrm{m}}^{\mathrm{T}}(\mathrm{t}) \mathrm{K} \Phi_{\mathrm{m}}(\mathrm{s})
$$

where $\mathrm{K}=\left(\mathrm{k}_{\mathrm{ij}}\right), \mathrm{i}, \mathrm{j}=1,2, \ldots, \mathrm{m}$ is the $\mathrm{m} \times \mathrm{m}$ block pulse coefficient matrix with

$$
\mathrm{k}_{\mathrm{ij}}=\frac{1}{\mathrm{~h}^{2}} \int_{0}^{1} \int_{0}^{1} \mathrm{k}(\mathrm{t}, \mathrm{s}) \phi_{\mathrm{i}}(\mathrm{t}) \phi_{\mathrm{j}}(\mathrm{s}) \mathrm{dtds} .(20)
$$

Theorem 3.2. [37] Suppose that $\mathrm{k}(\mathrm{t}, \mathrm{s}) \in \mathcal{L}^{2}([0,1) \times[0,1))$, and $\mathrm{e}(\mathrm{t}, \mathrm{s})=\mathrm{k}(\mathrm{t}, \mathrm{s})-\hat{\mathrm{k}}_{\mathrm{m}}(\mathrm{t}, \mathrm{s}),(\mathrm{t}, \mathrm{s}) \in \mathrm{D}$ which $\mathrm{D}=[0,1) \times[0,1)$ and $\hat{\mathrm{k}}(\mathrm{t}, \mathrm{s})=\sum_{\mathrm{i}=1}^{\mathrm{m}} \sum_{\mathrm{j}=1}^{\mathrm{m}} \mathrm{k}_{\mathrm{ij}} \phi_{\mathrm{i}}(\mathrm{t}) \phi_{\mathrm{j}}(\mathrm{s})$ is the block pulse series of $\mathrm{k}(\mathrm{t}, \mathrm{s})$. Then,

$$
\|\mathrm{e}(\mathrm{t}, \mathrm{s})\| \leq \frac{\mathrm{h}}{2 \sqrt{3}}\left(\sup _{(\mathrm{x}, \mathrm{y}) \in \mathrm{D}}\left|\mathrm{k}_{\mathrm{s}}^{\prime}(\mathrm{t}, \mathrm{s})\right|^{2}+\sup _{(\mathrm{x}, \mathrm{y}) \in \mathrm{D}}\left|\mathrm{k}_{\mathrm{t}}^{\prime}(\mathrm{t}, \mathrm{s})\right|^{2}\right)^{\frac{1}{2}}
$$

Lemma 3.3. Let $\mathrm{f}(\mathrm{t}) \simeq \mathrm{F}^{\mathrm{T}} \Phi_{\mathrm{m}}(\mathrm{t})$ then $[\mathrm{f}(\mathrm{t})]^{\mathrm{n}} \simeq \mathrm{F}_{\mathrm{n}}^{\mathrm{T}} \Phi_{\mathrm{m}}(\mathrm{t})$ where $\mathrm{F}_{\mathrm{n}}=\left(\mathrm{f}_{1}^{\mathrm{n}}, \mathrm{f}_{2}^{\mathrm{n}}, \ldots, \mathrm{f}_{\mathrm{m}}^{\mathrm{n}}\right)^{\mathrm{T}}$.

Lemma 3.4. For each function $h(x(t), u(t)) \in \mathbb{R}$, such that

$$
h(x(t), u(t))=\sum_{i=0}^{M} \sum_{j=0}^{N} h_{i j} x^{i}(t) u^{j}(t),(22)
$$

can be expand into BPFs as following form:

$$
\mathrm{h}(\mathrm{x}(\mathrm{t}), \mathrm{u}(\mathrm{t})) \simeq \Psi(\mathrm{X}, \mathrm{U}) \Phi_{\mathrm{m}}(\mathrm{t}),(23)
$$

where $\mathrm{X}$ and $\mathrm{U}$ are block pulse coefficients vectors of $\mathrm{x}(\mathrm{t})$ and $\mathrm{u}(\mathrm{t})$, respectively.

Proof: By the properties of BPFs and Lemma 3.3, we can obtain

$$
\begin{aligned}
h(x(t), u(t))= & \sum_{i=0}^{M} \sum_{j=0}^{N} h_{i j} x^{i}(t) u^{j}(t) \simeq \sum_{i=0}^{M} \sum_{j=0}^{N} h_{i j}\left(X_{i}^{T} \Phi_{m}(t)\right)\left(U_{j}^{T} \Phi_{m}(t)\right)^{T} \simeq \sum_{i=0}^{M} \sum_{j=0}^{N} h_{i j} X_{i}^{T} \frac{\widetilde{\mathrm{u}}_{j} \Phi_{m}(t)}{\Phi_{m}(t) \Phi_{m}(t)^{T} U_{j}} \\
& \simeq\left[\sum_{i=0}^{M} \sum_{j=0}^{N} h_{i j} X_{i}^{T} \widetilde{U}_{j}\right]_{1 \times m} \Phi_{m}(t) \simeq H_{m \times 1}^{T}(X, U) \Phi_{m}(t) .
\end{aligned}
$$

Remark 3.5. When function $\mathrm{h}(\mathrm{x}(\mathrm{t}), \mathrm{u}(\mathrm{t}))$ is strongly nonlinear, we can use bivariable Taylor series expansion of this function with respect to $\mathrm{x}(\mathrm{t})$ and $\mathrm{u}(\mathrm{t})$. Then above method can be applied easily by using operational matrices of integration and product.

\section{Solving stochastic optimal control of Volterra integral equation using BPFs}

Consider the following controlled stochastic Volterra integral equation: 


$$
x^{u}(t)=x_{0}+\int_{0}^{t} b\left(x^{u}(s), u(s)\right) d s+\int_{0}^{t} \sigma\left(x^{u}(s), u(s)\right) d B(s), x(0)=x_{0} .
$$

The aim of this section is to find the block pulse function coefficient of $x^{u}(t)$ and an admissible control function $\mathrm{u}(\mathrm{t})$ which minimizes the optimality criterion (2).

We expand $\mathrm{x}(\mathrm{t})$ and $\mathrm{u}(\mathrm{t})$ in terms of BPFs as:

$$
\begin{gathered}
\mathrm{x}(\mathrm{t}) \simeq \sum_{\mathrm{i}=1}^{\mathrm{m}} \mathrm{x}_{\mathrm{i}} \phi_{\mathrm{i}}(\mathrm{t})=\mathrm{X}^{\mathrm{T}} \Phi_{\mathrm{m}}(\mathrm{t}), \\
\mathrm{u}(\mathrm{t}) \simeq \sum_{\mathrm{i}=1}^{\mathrm{m}} \mathrm{u}_{\mathrm{i}} \phi_{\mathrm{i}}(\mathrm{t})=\mathrm{U}^{\mathrm{T}} \Phi_{\mathrm{m}}(\mathrm{t}) .(26)
\end{gathered}
$$

The expansion of $\mathrm{x}_{0}(\mathrm{t})$ in terms of BPFs is shown as follows:

$$
\mathrm{x}_{0} \simeq \mathrm{X}_{0}^{\mathrm{T}} \Phi_{\mathrm{m}}(\mathrm{t}) \cdot(27)
$$

According the Lemma 3.4 and Remark 3.5 we substitute (26) and (27) in equation (25)

$$
\begin{gathered}
\mathrm{X}^{\mathrm{T}} \Phi_{\mathrm{m}}(\mathrm{t}) \simeq \mathrm{X}_{0}^{\mathrm{T}} \Phi_{\mathrm{m}}(\mathrm{t})+\int_{0}^{\mathrm{t}} \mathrm{B}^{\mathrm{T}}(\mathrm{X}, \mathrm{U}) \Phi_{\mathrm{m}}(\mathrm{s}) \mathrm{ds}+\int_{0}^{\mathrm{t}} \Sigma^{\mathrm{T}}(\mathrm{X}, \mathrm{U}) \Phi_{\mathrm{m}}(\mathrm{s}) \mathrm{dB}(\mathrm{s}) \stackrel{(8),(9)}{\simeq} \mathrm{X}_{0}^{\mathrm{T}} \Phi_{\mathrm{m}}(\mathrm{t})+\mathrm{B}^{\mathrm{T}}(\mathrm{X}, \mathrm{U}) \mathrm{P}_{\mathrm{B}} \Phi_{\mathrm{m}}(\mathrm{t}) \\
+\Sigma^{\mathrm{T}}(\mathrm{X}, \mathrm{U}) \mathrm{P}_{\mathrm{BS}} \Phi_{\mathrm{m}}(\mathrm{t}) .
\end{gathered}
$$

Eq. (28), can be written as the following equation:

$$
\Psi^{\mathrm{T}}(\mathrm{X}, \mathrm{U}) \Phi_{\mathrm{m}}(\mathrm{t})=0,
$$

where $\Psi(\mathrm{X}, \mathrm{U}) \in \mathbb{R}^{\mathrm{m}}$. We can obtain a new equation $\Psi(\mathrm{X}, \mathrm{U})=0$ because the block pulse functions $\phi_{i}(t), i=1, \ldots, m, t \in[0,1)$ are linearly independent.

\subsection{Monte Carlo sampling and sample average approximation (SAA) method}

Monte Carlo simulation is a common method to reduce the scenario set which is based on random sampling. Higham used a Monte Carlo approach which is based on random variable are simulated with a random number generator and expected values are approximated by computed averages [38].We want to solve the problem without consideration of expected value with $\mathrm{N}$ discretized Brownian paths, so intend to estimate expected value with respect to underlying Brownian paths. Therefore, for each sample paths of Brownian motion, we have:

$$
J^{i}(X, U)=\int_{0}^{T} f^{i}\left(t, X^{T} \Phi_{m}(t), U^{T} \Phi_{m}(t)\right) d t+g^{i}\left(X^{T} \Phi_{m}(T)\right), \quad i=1,2, \ldots, N .(30)
$$

Now, the expectation function (2) is approximated by the sample average

$$
\mathrm{J}_{\mathrm{N}}(\mathrm{X}, \mathrm{U})=\frac{1}{\mathrm{~N}} \sum_{\mathrm{i}=1}^{\mathrm{N}} \mathrm{J}^{\mathrm{W}_{\mathrm{i}}}(\mathrm{X}, \mathrm{U}) .(31
$$

For notational simplicity, we use $\mathrm{J}(\mathrm{X}, \mathrm{U})$ instead of $\mathrm{J}^{\mathrm{i}}(\mathrm{X}, \mathrm{U})$. The problem minimizes the optimality 
criterion (30) to find $\mathrm{X}$ and $\mathrm{U}$ with constraints set $\Psi^{\mathrm{T}}(\mathrm{X}, \mathrm{U})=0$.

Consider the following problem:

$$
\left\{\begin{array}{c}
\boldsymbol{P} \text { : Minimize } \mathrm{J}(\mathrm{X}, \mathrm{U}) \\
\text { s.t. } \Psi(\mathrm{X}, \mathrm{U})=0
\end{array}\right.
$$

The necessary optimality conditions can be stated as follows. If $(\bar{X}, \bar{U})$ is an optimal solution $P$, under a suitable constraint, there exist a vector $\lambda=\left(\lambda_{1}, \lambda_{2}, \ldots, \lambda_{\mathrm{m}}\right)^{\mathrm{T}}$ such that:

$$
\bar{J}(X, U, \lambda)=J(X, U)+\lambda^{T} \Psi(X, U)
$$

where $\lambda$ is Lagrange multiplier associated with the constraint $\Psi(\mathrm{X}, \mathrm{U})=0$. Then, we have

$$
\begin{gathered}
\frac{\partial \overline{\mathrm{J}}}{\partial \mathrm{X}_{\mathrm{i}}}(\mathrm{X}, \mathrm{U}, \lambda)=0, \\
\frac{\partial \overline{\mathrm{J}}}{\partial \mathrm{U}_{\mathrm{i}}}(\mathrm{X}, \mathrm{U}, \lambda)=0, \\
\frac{\partial \overline{\mathrm{J}}}{\partial \lambda_{\mathrm{i}}}(\mathrm{X}, \mathrm{U}, \lambda)=0,
\end{gathered}
$$

for $\mathrm{i}=1,2, \ldots, \mathrm{m}$.

\subsection{Special Case}

In this section, we assume that the state of the system is generated by a linear noisy system

$$
\mathrm{x}^{\mathrm{u}}(\mathrm{t})=\mathrm{x}_{0}+\int_{0}^{\mathrm{t}} \mathrm{Ax}{ }^{\mathrm{u}}(\mathrm{s}) \mathrm{d} \mathrm{s}+\int_{0}^{\mathrm{t}} \mathrm{Bu}(\mathrm{s}) \mathrm{d} \mathrm{s}+\int_{0}^{\mathrm{t}} \mathrm{dB}(\mathrm{s})
$$

where $\mathrm{B}(\mathrm{s})$ is a Brownian motion. Also, consider the performance functional

$$
J(u)=E\left[\int_{0}^{T} Q x^{u}(s)^{2} d s+\int_{0}^{T} R u^{2}(s) d s+Q_{T} x^{u}(T)^{2}\right]
$$

The action space considered by the controller is assumed to be the set of linear feedback actions. This means that the optimal control strategy is a function of the time and the current state. The set of this linear feedback actions also provides an optimal solution for the stochastic control problem (35),(36).

Theorem 4.1.[39] Assume that the Riccati differential equation

$$
\dot{\mathrm{k}}(\mathrm{t})=-2 \mathrm{Ak}(\mathrm{t})+\mathrm{Sk}^{2}(\mathrm{t})-\mathrm{Q}, \mathrm{k}(\mathrm{T})=\mathrm{Q}_{\mathrm{T}},
$$


has a solution on $[0, T)$, where $S=R^{-1} B^{2}$. Then the control $u_{l f b}(t)=-R^{-1} B k(t) X(t)$ is optimal for the stochastic control problem

$$
\min _{u(t) \in \Gamma^{I f b}} E\left[\int_{0}^{T}\left(Q x^{u}(t)^{2}+R F^{2}(t) x^{u}(t)^{2}\right) d t+Q_{T} x^{u}(T)^{2}\right]
$$

subject to the system (35), where

$$
\Gamma^{\mathrm{lfb}}=\{\mathrm{u}(\mathrm{t}) \mid \mathrm{u}(\mathrm{t})=\mathrm{F}(\mathrm{t}) \mathrm{x}(\mathrm{t})\},
$$

and $\mathrm{F}($.$) is a piecewise continuous function.$

\section{Numerical examples}

To demonstrate the applications of the both presented methods, open loop and linear feedback control, we consider the following examples.

Example 5.1 Consider the following performance functional and dynamical system:

$$
\begin{gathered}
J=\min _{u \in U} E\left\{\frac{1}{2} \int_{0}^{1}\left[2 x^{2}(t)+u(t)\right] d t\right\},(40) \\
d x(t)=\left(-\frac{1}{2} x(t)+u(t)\right) d t+d B(t), x(0)=1
\end{gathered}
$$

It is clear that we can write the following Volterra integral equation instead of Eq.(41):

$$
\int_{0}^{t} d x(s)=\int_{0}^{t}\left(-\frac{1}{2} x(s)+u^{2}(s)\right) d t+\int_{0}^{t} d B(s) \cdot(42)
$$

\section{Open Loop Control:}

By substituting Eq. (26) and Eq. (27) in Eq. (42) and (40), we have

$$
\begin{aligned}
\mathrm{x}(\mathrm{t})-\mathrm{x}(0) \simeq \mathrm{X}^{\mathrm{T}} & \Phi_{\mathrm{m}}(\mathrm{t})-\mathrm{X}_{0}^{\mathrm{T}} \Phi_{\mathrm{m}}(\mathrm{t}) \simeq \int_{0}^{\mathrm{t}}\left(-\frac{1}{2} \mathrm{X}^{\mathrm{T}} \Phi_{\mathrm{m}}(\mathrm{s})+\mathrm{U}^{\mathrm{T}} \Phi_{\mathrm{m}}(\mathrm{s})\right) \mathrm{ds}+\int_{0}^{\mathrm{t}} 1^{\mathrm{T}} \Phi_{\mathrm{m}}(\mathrm{s}) \mathrm{dB}(\mathrm{s}) \\
\simeq & \frac{1}{2} \mathrm{X}^{\mathrm{T}} \int_{0}^{\mathrm{t}} \Phi_{\mathrm{m}}(\mathrm{s}) \mathrm{d} s+\mathrm{U}^{\mathrm{T}} \int_{0}^{\mathrm{t}} \Phi_{\mathrm{m}}(\mathrm{s}) \mathrm{ds}+1^{\mathrm{T}} \int_{0}^{\mathrm{t}} \Phi_{\mathrm{m}}(\mathrm{s}) \mathrm{dB}(\mathrm{s}) \stackrel{(8),(9)}{\simeq}-\frac{1}{2} \mathrm{X}^{\mathrm{T}} \mathrm{P}_{\mathrm{B}} \Phi_{\mathrm{m}}(\mathrm{t})+\mathrm{U}^{\mathrm{T}} \mathrm{P}_{\mathrm{B}} \Phi_{\mathrm{m}}(\mathrm{t}) \\
& +1^{\mathrm{T}} \mathrm{P}_{\mathrm{BS}} \Phi_{\mathrm{m}}(\mathrm{t}),
\end{aligned}
$$

and 


$$
\begin{aligned}
\mathrm{J}(\mathrm{X}, \mathrm{U})=\frac{1}{2} \int_{0}^{1} 2(\mathrm{X}^{\mathrm{T}} \overbrace{\Phi_{\mathrm{m}}(\mathrm{t})\left(\Phi_{\mathrm{m}}^{\mathrm{T}}(\mathrm{t}) \mathrm{X}\right.}^{\operatorname{diag}(\mathrm{t}) \Phi_{\mathrm{m}}(\mathrm{t})}) \mathrm{dt}+\int_{0}^{1} \frac{1}{2}(\mathrm{U}^{\mathrm{T}} \underbrace{\Phi_{\mathrm{m}}(\mathrm{t}) \Phi_{\mathrm{m}}^{\mathrm{T}}(\mathrm{t}) \mathrm{U}}_{\operatorname{diag}(\mathrm{U}) \Phi_{\mathrm{m}}(\mathrm{t})}) d \mathrm{t} \\
\simeq\left(\mathrm{X}^{\mathrm{T}} \operatorname{diag}(\mathrm{X})+\frac{1}{2} \mathrm{U}^{\mathrm{T}} \operatorname{diag}(\mathrm{U})\right)\left(\int_{0}^{1} \Phi_{\mathrm{m}}(\mathrm{t}) \mathrm{dt}\right) \simeq \quad\left(\mathrm{X}^{\mathrm{T}} \operatorname{diag}(\mathrm{X})+\frac{1}{2} \mathrm{U}^{\mathrm{T}} \operatorname{diag}(\mathrm{U})\right)
\end{aligned}
$$

where $1=(1,1, \ldots, 1)_{\mathrm{m} \times 1}^{\mathrm{T}}$. Also, we obtain:

$$
\Psi^{\mathrm{T}}(\mathrm{X}, \mathrm{U})=\mathrm{X}^{\mathrm{T}}-\mathrm{X}_{0}^{\mathrm{T}}+\frac{1}{2} \mathrm{X}^{\mathrm{T}} \mathrm{P}_{\mathrm{B}}-\mathrm{U}^{\mathrm{T}} \mathrm{P}_{\mathrm{B}}-1^{\mathrm{T}} \mathrm{P}_{\mathrm{BS}},(45)
$$

and

$$
\mathrm{J}(\mathrm{X}, \mathrm{U})=\mathrm{h}\left(\mathrm{X}^{\mathrm{T}} \operatorname{diag}(\mathrm{X})+\frac{1}{2} \mathrm{U}^{\mathrm{T}} \operatorname{diag}(\mathrm{U})\right) 1
$$

\section{Linear Feedback Control}

By using Theorem 4.1, the linear feedback optimal control is

$$
\mathrm{u}_{\mathrm{lfb}}(\mathrm{t})=\mathrm{k}(\mathrm{t}) \mathrm{x}(\mathrm{t}),(47)
$$

where

$$
\mathrm{k}(\mathrm{t})=\frac{-2\left(\mathrm{e}^{-3 \mathrm{t}+3}-1\right)}{1+2 \mathrm{e}^{-3 \mathrm{t}+3}}
$$

By terms of approximating $\mathrm{k}(\mathrm{t})$ respect to the BPFs and substituting the obtained formed into (40) and (41), the numerical results are shown in Tables 1 and 2 . The graphs of approximate solutions computed by the both presented methods are given in Fig.1.
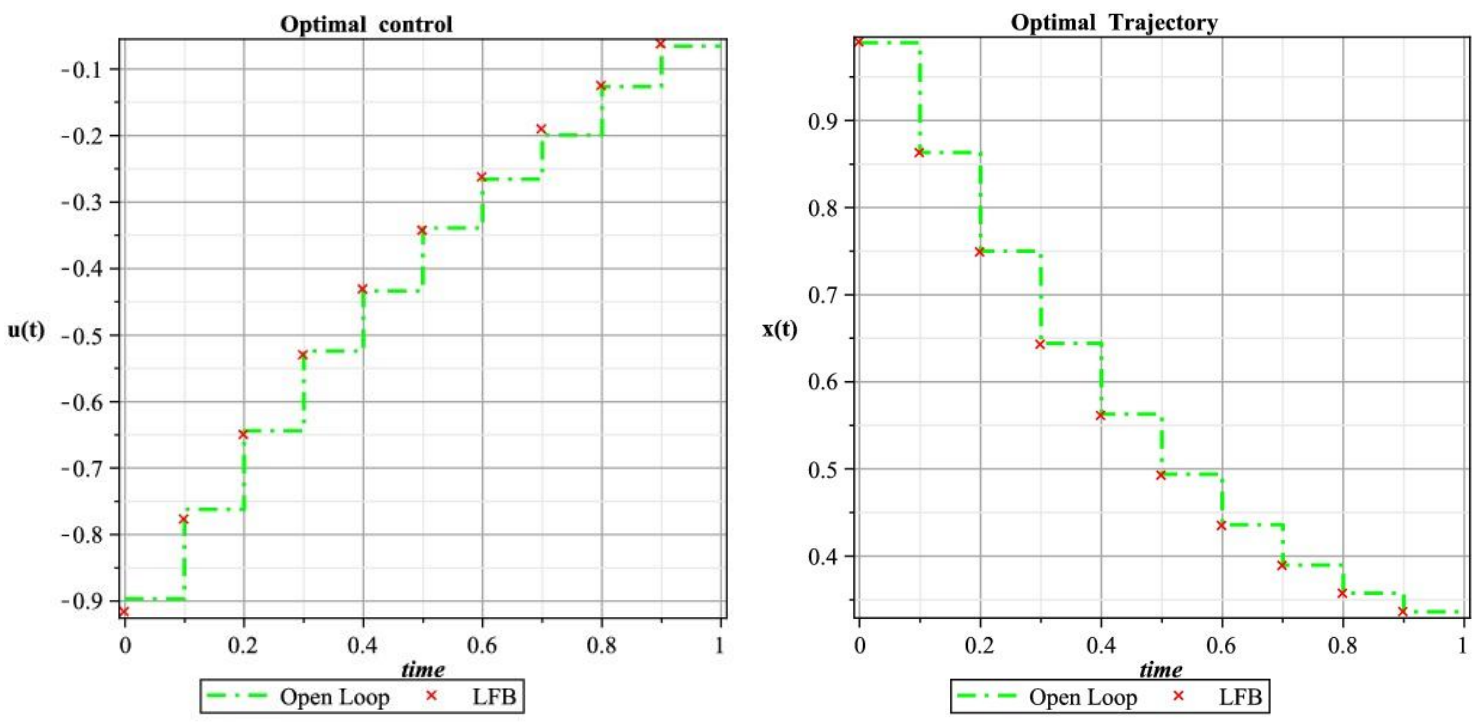

Fig 1. Graphs of approximate solutions for Example 5.1. 
Table 1. Open loop and linear feedback control in different times for Example 5.1.

\begin{tabular}{ccccc}
\hline $\mathrm{t}$ & $\mathrm{X}_{\text {Open loop }}(\mathrm{t})$ & $\mathrm{U}_{\text {Open loop }}(\mathrm{t})$ & $\mathrm{X}_{\mathrm{LFB}}(\mathrm{t})$ & $\mathrm{U}_{\text {LFB }}(\mathrm{t})$ \\
\hline 0 & 0.9892287 & -0.8962915 & 0.9891420 & -0.9170710 \\
0.1 & 0.8632260 & -0.7620760 & 0.8620300 & -0.7779690 \\
0.2 & 0.7500150 & -0.6440920 & 0.7480970 & -0.6507030 \\
0.3 & 0.6443140 & -0.5242450 & 0.6420490 & -0.5309350 \\
0.4 & 0.5628039 & -0.4333895 & 0.5604060 & -0.4320720 \\
0.5 & 0.4940540 & -0.3388410 & 0.4918140 & -0.3437310 \\
0.6 & 0.4360210 & -0.2657610 & 0.4339930 & -0.2635810 \\
0.7 & 0.3897110 & -0.1993660 & 0.3882690 & -0.1914860 \\
0.8 & 0.3572560 & -0.1263700 & 0.3563050 & -0.1261470 \\
0.9 & 0.3360800 & -0.0655266 & 0.3353020 & -0.0634211 \\
\hline
\end{tabular}

Table 2. Difference between Open loop and linear feedback methods for Example 5.1.

\begin{tabular}{ccc}
\hline $\mathrm{t}$ & $\mathrm{e}_{\mathrm{x}}$ & $\mathrm{e}_{\mathrm{u}}$ \\
\hline 0 & $8.67080 \mathrm{E}-5$ & $2.07794 \mathrm{E}-2$ \\
0.1 & $1.19600 \mathrm{E}-3$ & $1.58930 \mathrm{E}-2$ \\
0.2 & $1.91800 \mathrm{E}-3$ & $6.61100 \mathrm{E}-3$ \\
0.3 & $2.26500 \mathrm{E}-3$ & $6.69000 \mathrm{E}-3$ \\
0.4 & $2.39791 \mathrm{E}-3$ & $1.31746 \mathrm{E}-3$ \\
0.5 & $2.24000 \mathrm{E}-3$ & $4.89000 \mathrm{E}-3$ \\
0.6 & $2.02800 \mathrm{E}-3$ & $2.18000 \mathrm{E}-3$ \\
0.7 & $1.44200 \mathrm{E}-3$ & $7.88000 \mathrm{E}-3$ \\
0.8 & $9.51000 \mathrm{E}-4$ & $2.23000 \mathrm{E}-4$ \\
0.9 & $7.78000 \mathrm{E}-4$ & $2.10550 \mathrm{E}-3$ \\
\hline
\end{tabular}


Example 5.2 Consider the following performance functional and dynamical system:

$$
\begin{aligned}
& J=\min _{u \in U} E\left\{\frac{1}{2}\left[\int_{0}^{1} u^{2}(t) d t+x^{2}(1)\right]\right\}, \\
& d x(t)=u(t) d t+d B(t), x(0)=1 .
\end{aligned}
$$

The numerical results for the both presented methods are shown in Tables 3 and 4. The graphs of approximate solutions computed in Fig. 2.
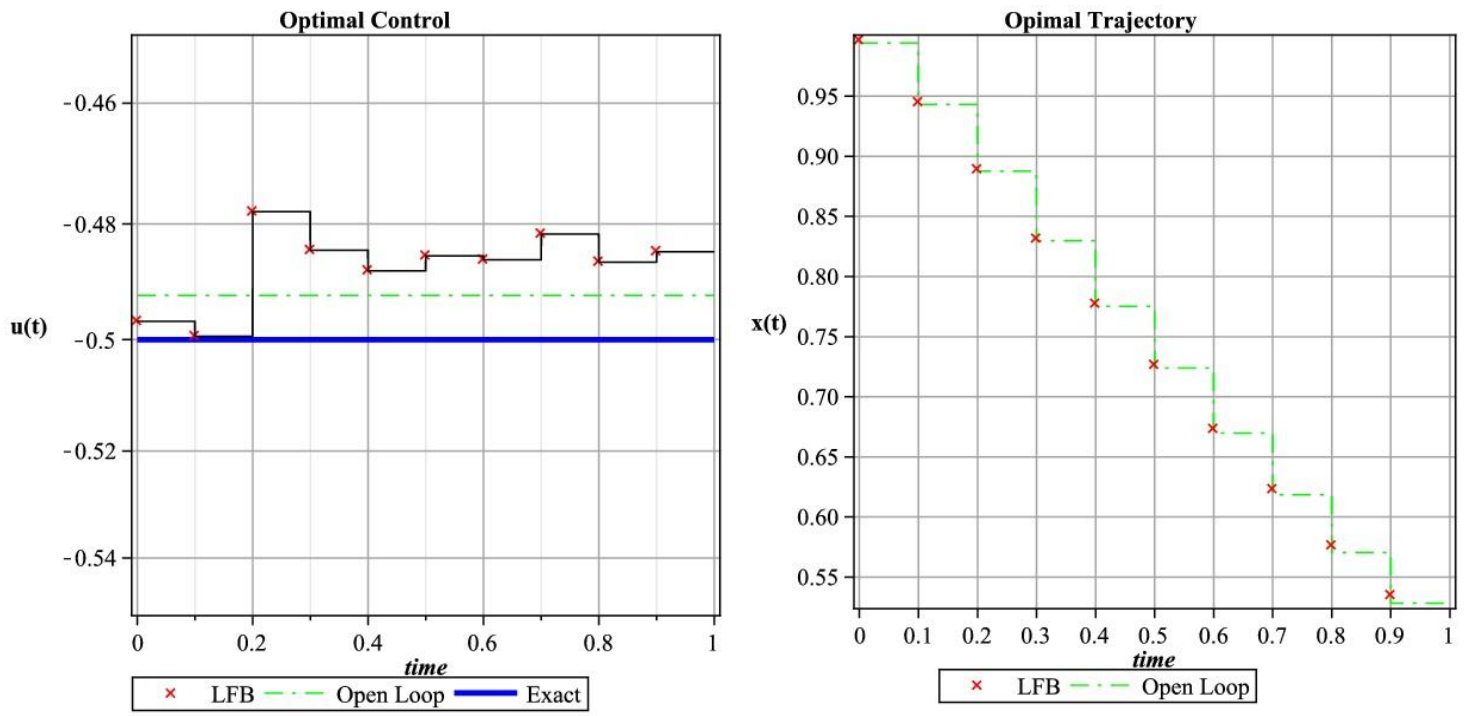

Fig 2. Graphs of approximate solutions for Example 5.2. 
Table 3.Open loop and linear feedback control in different times for Example 5.2.

\begin{tabular}{ccccc}
\hline $\mathrm{t}$ & $\mathrm{X}_{\text {Open loop }}(\mathrm{t})$ & $\mathrm{U}_{\text {Open loop }}(\mathrm{t})$ & $\mathrm{X}_{\mathrm{LFB}}(\mathrm{t})$ & $\mathrm{U}_{\mathrm{LFB}}(\mathrm{t})$ \\
\hline 0 & 0.9962740 & -0.4932620 & 0.9935670 & -0.4967835 \\
0.1 & 0.9451660 & -0.4932620 & 0.9489053 & -0.4994239 \\
0.2 & 0.8894460 & -0.4932620 & 0.8603350 & -0.4779639 \\
0.3 & 0.8314370 & -0.4932640 & 0.8236622 & -0.4845072 \\
0.4 & 0.7768210 & -0.4932660 & 0.7807941 & -0.4879963 \\
0.5 & 0.7252890 & -0.4932660 & 0.7281781 & -0.4854521 \\
0.6 & 0.6711330 & -0.4932660 & 0.6805939 & -0.4861385 \\
0.7 & 0.6197070 & -0.4932640 & 0.6262345 & -0.4817189 \\
0.8 & 0.5715320 & -0.4932620 & 0.5838139 & -0.4865116 \\
0.9 & 0.5294100 & -0.4932620 & 0.5331870 & -0.4847154 \\
\hline
\end{tabular}

Table 4. Difference between Open loop and linear feedback methods for Example 5.2.

\begin{tabular}{ccc}
\hline $\mathrm{t}$ & $\mathrm{e}_{\mathrm{x}}$ & $\mathrm{e}_{\mathrm{u}}$ \\
\hline 0 & $2.70700 \mathrm{E}-3$ & $3.52150 \mathrm{E}-3$ \\
0.1 & $3.73930 \mathrm{E}-3$ & $6.16190 \mathrm{E}-3$ \\
0.2 & $2.91110 \mathrm{E}-2$ & $1.52981 \mathrm{E}-2$ \\
0.3 & $7.77480 \mathrm{E}-3$ & $8.75680 \mathrm{E}-3$ \\
0.4 & $3.97310 \mathrm{E}-3$ & $5.26970 \mathrm{E}-3$ \\
0.5 & $2.88910 \mathrm{E}-3$ & $7.81390 \mathrm{E}-3$ \\
0.6 & $9.46090 \mathrm{E}-3$ & $7.12750 \mathrm{E}-3$ \\
0.7 & $6.52750 \mathrm{E}-3$ & $1.15451 \mathrm{E}-2$ \\
0.8 & $1.22819 \mathrm{E}-2$ & $6.75040 \mathrm{E}-3$ \\
0.9 & $3.77700 \mathrm{E}-3$ & $8.54660 \mathrm{E}-3$ \\
\hline
\end{tabular}

\section{Conclusion}

In this paper a numerical method, which is based on block pulse functions, is developed to calculate both open loop and feedback optimal controls of stochastic Volterra integral equations. There is no 
analytical solution for forgoing stochastic optimal control problems. In this paper the numerical method for solving general case of these problems was proposed. For future work this method can be solve by other orthogonal functions or polynomials, such as Hybrid functions, Chebyshev polynomials and Spline polynomials.

\section{References}

[1] A. W. Heemink, I. D. M. Metzelaar, Data assimilation into a numerical shallow water flow model: A stochastic optimal control approach, J. Marine. Syst. 6 (1995) 145-158.

[2] Y. Shastria, U. Diwekarb, Sustainable ecosystem management using optimal control theory: Part 2 (stochastic systems), J. Theor. Biol. 241 (2006) 522-532.

[3] A. J. Coldman, J. M. Murray, Optimal control for a stochastic model of cancer chemotherapy, Math. Biosci. 168 (2000) 187-200.

[4] J. L. Stein, Applications of stochastic optimal control/dynamic programming to international finance and debt crises, Nonlinear. Anal. 63 (2005) e2033-e2041.

[5] J. M. Petersen, M. A. Petersen, Bank management via stochastic optimal control, Automatica. 42 (2006) 1395-1406.

[6] S. U. Acikgoz, U. M. Diwekar, Blood glucose regulation with stochastic optimal control for insulindependent diabetic patients, Chem. Eng. Sci. 65 (2010) 1227-1236.

[7] P. T. Benavides, U. Diweka, Optimal control of biodiesel production in a batch reactor Part II: Stochastic control, Fuel. 94 (2012) 218-226.

[8] W. H. Fleming, C. J. Rishel, Deterministic and Stochastic Optimal Control, Springer-Verlag, 1975.

[9] W. H. Fleming, H. M. Soner, Controlled Markov Processes and Viscosity Solutions, Springer, 2006.

[10] R. E. Bellman, Dynamic Programming, Princeton University Press, Princeton, NJ, 1957.

[11] R. E. Bellman, S. E. Dreyfus, Applied Dynamic Programming, Princeton University Press, Princeton, NJ, 1962.

[12] D. E. Kirk, Optimal Control Theory An Introduction, Prentice-Hall, Englewood Cliffs, 1970.

[13] A. B.Pantelev, A. C. Bortakovski, T. A. Letova, Some Issues and Examples in Optimal Control, MAI Press, Moscow (in Russian), 1996.

[14] E. R. Pinch, Optimal Control and the Calculus of Variations, Oxford University Press, London, 1993.

[15] L. S. Pontryagin, The Mathematical Theory of Optimal Processes, Interscience, John Wiley and Sons, 1962. 
[16] A. Jajarmi, N. Pariz, S. Effati, A. V. Kamyad, Infinite horizon optimal control for nonlinear interconnected Large-Scale dynamical systems with an application to optimal attitude control, Asian. J. Control. 15 (2013) 1-12.

[17] B. Kafash, A. Delavarkhalafi, S. M. Karbassi, Application of variational iteration method for Hamilton-Jacobi-Bellman equations, Appl. Math. Modell. 37 (2013) 3917-3928.

[18] H. M. Jaddu, Direct solution of nonlinear optimal control problems using quasilinearization and Chebyshev polynomials, J. Franklin Inst. 339 (2002) 479-498.

[19] B. Kafash, A. Delavarkhalafi, S. M. Karbassi, Application of Chebyshev polynomials to derive efficient algorithms for the solution of optimal control problems, Sci. Iran. 19 (2012) 795-805.

[20] K. Maleknejad, H. Almasieh, Optimal control of Volterra integral equations via triangular functions, Math. Comput. Modell. 53 (2011) 1902-1909.

[21] H. R. Erfanian, M. H. Noori Skandari, Optimal control of an HIV model, The Journal of Mathematics and Computer Science Vol .2 No.4 (2011) 650-658.

[22] E. Hesameddini, A. Fakharzadeh Jahromi, M. Soleimanivareki, H. Alimorad, Differential transformation method for solving a class of nonlinear optimal control problems, The Journal of Mathematics and Computer Science Vol .5 No.1 (2012) 67-74.

[23] C. Myers, Stochastic Control, Sciyo, Croatia, 2010.

[24] B. Øksendal, T. Zhang, Optimal control with partial information for stochastic Volterra equations, Int. J. Stoch. Anal. (2010) Art. ID 329185, 25 pp.

[25] S. Ji, X. Y. ZHOU, A maximum principle for stochastic optimal control with terminal state constraints and its applications, Commun. Info. Sys. 6 (2006) 321-338.

[26] Z. Wu, A general maximum principle for optimal control of forward-backward stochastic systems, Automatica. 49 (2013) 1473-1480.

[27] S. Bonaccorsi, F. Confortola, E. Matrogiacomo, Optimal control for stochastic Volterra equations with completely monotone kernels, Siam. J. Control. Optim. 50 (2012) 748-789.

[28] N. Kuchkina, L. Shaikhet, Optimal control of Volterra Type stochastic difference equations, Comput. Math. Appl. 36 (1998) 251-259.

[29] H. J. Kushner, Numerical methods for stochastic control problems in finance, Lefschetz Center for Dynamical Systems and Center for Control Sciences, Division of Applied Mathematics, Brown University, 1995.

[30] C. Munk, Numerical methods for continuous-time, continuous-state stochastic control problems, Publications from department of management 97, No. 11, 1997.

[31] C. Munk, The Markov chain approximation approach for numerical solution of stochastic control problems: experiences from Merton's problem, Appl. Math. Comput. 136 (2003) 47-77. 
[32] W. Chavanasporn, C. O. Ewald, A numerical method for solving stochastic optimal control problems with linear control, Comput. Econ. 39 (2012) 429-446.

[33] K. Maleknejad, M. Khodabin, M. Rostami, Numerical solution of stochastic Volterra integral equations by a stochastic operational matrix based on block pulse functions, Math. Comput. Modell. 55 (2012) 791-800.

[34] G. P. Rao, Piecewise constant orthogonal functions and their application to systems and control, Springer, Berlin, 1983.

[35] Z.H. Jiang, W. Schaufelberger, Block pulse functions and their applications in control systems, Springer-Verlag, 1992.

[36] K. Maleknejad, M. Khodabin, M. Rostami, A numerical method for solving m-dimensional stochastic Itô-Volterra integral equations by stochastic operational matrix, Comput. Math. Appl. 63 (2012) 133-143.

[37] M. Khodabin, K. Maleknejad, M. Rostami, M. Nouri, Numerical approach for solving stochastic Volterra-Fredholm integral equations by stochastic operational matrix, Comput. Math. Appl. 64 (2012) 1903-1913.

[38] D. J. Higham, An algorithmic introduction to numerical simulation of stochastic differential equations, Siam Rev. 43 (2001) 525-546.

[39] J. Engwerda, LQ Dynamic Optimization and Diffrential Games, John Wileyn and Sons LTD, 2005. 